\title{
150 kW Class Solar Electric Propulsion Spacecraft Power Architecture Model
}

\author{
Jeffrey T. Csank ${ }^{1}$, Michael V. Aulisio ${ }^{2}$ \\ NASA Glenn Research Center, Cleveland, OH, 44135, USA \\ and \\ Benjamin Loop ${ }^{3}$ \\ PC Krause and Associates, Inc., 3000 Kent Ave, Suite C1-100, West Lafayette, IN 47906, USA
}

The National Aeronautics and Space Administration (NASA) Solar Electric Propulsion Technology Demonstration Mission (SEP TDM), in conjunction with PC Krause and Associates, has created a Simulink-based power architecture model for a 50 kilo-Watt $(\mathrm{kW})$ solar electric propulsion system. NASA has extended this model to investigate $150 \mathrm{~kW}$ solar electric propulsion systems. Increasing the power capability to $150 \mathrm{~kW}$ is an intermediate step to the anticipated power requirements for Mars and other deep space applications. The highpower solar electric propulsion capability has been identified as a critical part of NASA's future beyond-low-Earth-orbit for human-crewed exploration missions. This paper presents four versions of a $150 \mathrm{~kW}$ architecture, simulation results, and a discussion of their merits.

\section{Nomenclature}

$\begin{array}{ll}\text { AEPS } & \text { Advanced Electric Propulsion System } \\ \text { ARRM } & \text { Asteroid Redirect Robotic Mission } \\ \text { DDCU } & \text { DC-DC Converter Unit } \\ \text { DSG } & \text { Deep Space Gateway } \\ \text { HET } & \text { Hall Effect Thrusters } \\ \text { IPS } & \text { Ion Propulsion System } \\ \text { ISS } & \text { International Space Station } \\ \text { PDU } & \text { Power Distribution Unit } \\ \text { PPB } & \text { Power and Propulsion Bus } \\ \text { PPU } & \text { Power Processor Unit } \\ \text { RBI } & \text { Remote Bus Interrupter } \\ \text { SA } & \text { Solar Array } \\ \text { SEP } & \text { Solar Electric Propulsion } \\ \text { SEPM } & \text { Solar Electric Propulsion Module } \\ \text { SSU } & \text { Sequential Shunt Unit }\end{array}$

\section{Introduction}

$\mathrm{T}$ HE National Aeronautics and Space Administration (NASA), in conjunction with PC Krause and Associates, has created a power architecture model for a $50 \mathrm{~kW}$ class Government reference design of the Solar Electric Propulsion Module (SEPM) of the Asteroid Redirect Robotic Mission (ARRM). ${ }^{1}$ This vehicle and power system was originally designed to power a high-power solar electric propulsion (SEP) system to return an asteroidal mass for rendezvous and characterization in a companion human-crewed mission. NASA is also evaluating applicability of a similar SEP system to that of the power and propulsion bus (PPB) of the Deep Space Gateway (DSG). The DSG will

${ }^{1}$ Electrical Engineer, Power Management and Distribution Branch, jeffrey.t.csank@nasa.gov, AIAA Sr. Member

2 Electrical Engineer, Power Management and Distribution Branch, michael.v.aulisio@nasa.gov

${ }^{3}$ Director of Engineering Services, PC Krause and Associates, loop@pcka.com

American Institute of Aeronautics and Astronautics 
be used to perform missions in cislunar space, and the PPB will be used for station keeping and the ability to transfer among a family of orbits in the lunar vicinity to enable a variety of missions (surface of the moon, departure from high lunar orbit to other destinations in the solar system). ${ }^{5}$ As an extension, NASA is investigating higher power architectures for missions applicable to deep space transportation, such as to Mars. Higher-Power SEP is enabling for these deep space missions as it is necessary to minimize the time spent in transportation from cislunar space to the Mars surface.

The $150 \mathrm{~kW}$ power system uses an architecture similar to the $50 \mathrm{~kW}$ system as the foundation for the model. Propulsion strings of $12.5 \mathrm{~kW}$ are implemented as basic building blocks. Flight versions of these electric propulsion strings are being developed under the NASA SEP TDM-led Advanced Electric Propulsion System (AEPS) Project. The power system architecture consists of twelve propulsion strings, providing roughly $150 \mathrm{~kW}$ of power for propulsion, and two additional $2.5 \mathrm{~kW}$ branch switches for distributing power to avionics and other vehicle support systems totaling $155 \mathrm{~kW}$ of power. Each propulsion string includes a power processor unit (PPU), which converts the solar array generated power into the various regulated currents and voltages required to start and operate the Hall Effect Thrusters (HET).

This paper compares four different architectures. The first architecture contains two solar arrays connected to a single bus which distributes power to twelve propulsion strings and two avionics and support strings. The second architecture is a segmented system in which a solar array powers a single bus which powers six power strings and a single avionics and support system string. The remaining two architectures are regulated versions of the first two architectures where a sequential shunt unit is added between the solar array and the bus to regulate the voltage to the bus by adding a simulated load to the system. A simulation study illustrates the feasibility of each system operating under multiple conditions. The simulations have been developed in the MATLAB ${ }^{\circledR} /$ Simulink ${ }^{\circledR}$, the MathWorks Inc., environment using a custom Simulink International Space Station (ISS) model library developed by PC Krause and Associates, Inc. The ISS model library contains MATLAB functions and Simulink blocks to represent the salient performance characteristics of the ISS electrical power system to support the development of intelligent energy management controls. ${ }^{2}$ In particular, the model library is capable of facilitating stability analysis through its prediction of transient effects. Both large signal and small signal stability effects can be evaluated (see, for example, Section II$\mathrm{C}$ below). The basic structure of the SEP spacecraft power architecture Simulink model consists of solar array blocks, from the ISS library, connected to one or more loads, including both the propulsion strings and support power. Each propulsion power string is designated to draw $12.5 \mathrm{~kW}$ at $100 \mathrm{Vdc}$. For this study, it is assumed that there will be two solar arrays each capable of providing approximately $75-80 \mathrm{~kW}$ of power and has an irradiance of $1.380 \mathrm{~kW} / \mathrm{m}^{2}$ at any moment in time. The solar array is configured with 210 cells in each string, 310 parallel strings to deliver the required power at the appropriate voltage level. In steady-state and full load, each solar array provides $79.4 \mathrm{~kW}$ of power at $117.8 \mathrm{~V}$ and 673.95 amperes.

For electric propulsion vehicles, electric power is required for both driving the vehicle and powering all the avionics and vehicle support systems. Therefore the vehicle requires high availability of electrical power. When considering some of the possible support systems, such as life support for manned space vehicles, continuous operation of these systems is critical. The unanticipated interruption of electric power availability is often the result of some component failure, or fault, in the system. The goal is to design the system to be fault tolerant, or be able to withstand a single failure without major consequence. Increasing the fault tolerance, or availability, usually requires increasing the cost and complexity of the system. Taking advantage of partitioning and redundancy can help achieve fault tolerance. Redundancy is adding additional components to the system to replace the failed components, whereas partitioning ensures that a failure in one subsystem will not impact the other subsystems. ${ }^{3}$

\section{150kW Non-Segmented Power System}

The $150 \mathrm{~kW}$ class non-segmented power system consists of twelve electric propulsion strings consuming $12.5 \mathrm{~kW}$ of power with two additional branches dedicated for $2.5 \mathrm{~kW}$ of power each for avionics and other support systems. This architecture features a common-bus which is supplied power from two solar arrays (SA-1, SA-2) and distributes the $155 \mathrm{~kW}$ of power to the fourteen branches, reference Figure 1. Each propulsion string is connected to the solar array (or bus) through a Remote Bus Interrupter (RBI) block, which acts as a switch and is either open or closed. In Simulink, each propulsion string is represented by a constant power DC-DC Converter Unit (DDCU), which converts power from the source to the load at the desired voltages, connected to a constant power load. The DDCU and constant power load for each propulsion string is configured to represent the Ion Propulsion System (IPS) Power Processing Unit (PPU) function and power demand. The avionics and support power loads consist of a DDCU connected to a constant power load at $100 \mathrm{~V}$ and another DDCU which is connected to a constant power load at $30 \mathrm{~V}$, which models the loads attached to a $100 \mathrm{~V}$ Power Distribution Unit (PDU) and $30 \mathrm{~V}$ PDU. At full power the solar arrays provide

American Institute of Aeronautics and Astronautics 
the bus with $158.83 \mathrm{~kW}$ of total power including losses. This likely represents the simplest architecture of one such class system and is presented as a starting point in this feasibility study.

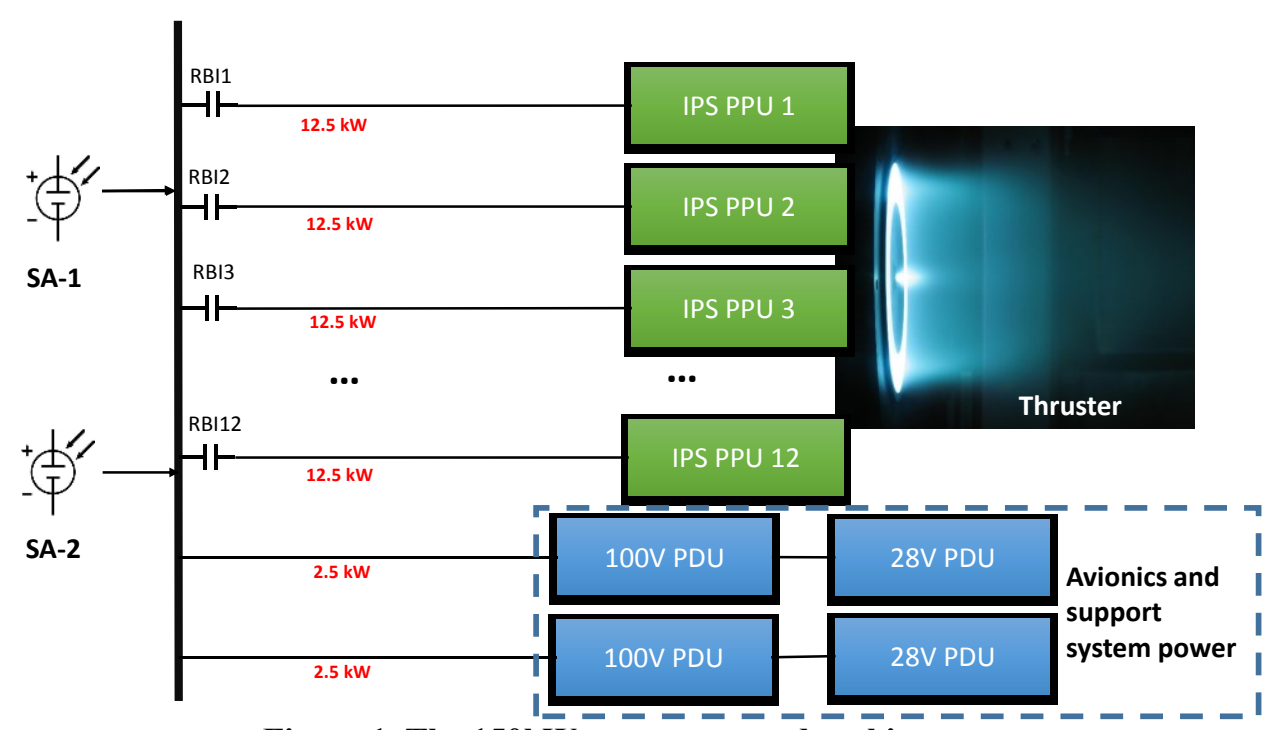

Figure 1. The 150kW non-segmented architecture.

\section{A. Transient Response}

Consider turning all twelve propulsion power strings on at exactly the same time. Before the power is turned on, the solar arrays should be at or near the solar array open circuit voltage, which is approximately 136 volts, and only providing power to the two $2.5 \mathrm{~kW}$ avionics and support system strings. At a simulation time of 4 seconds, a single step command is given to command all the propulsion string loads to turn on at the same time. When this occurs, the current out of and power to the bus from the solar arrays increases and the solar array voltages decrease. The solar array voltage for SA-1 (top plot) and total power supplied by the array and total power consumed by the loads (bottom plot) is shown in Figure 2. Figure 2 shows that when the loads are powered on the power system goes through a short transient, approximately 3 milliseconds, and the solar array settles to a lower voltage at 46 volts and produces approximately $80 \mathrm{~kW}$ of power, which is less than the designed $155 \mathrm{~kW}$ of power. The existence and stability issues regarding this low-voltage/low-power state is discussed in more detail in Section C below.

To decrease the instantaneous demand on the generation and distribution systems, a 1 millisecond delay is inserted between the commands to each RBI and provide power to each propulsion string incrementally. The solar array voltage and total power both generated and consumed, is shown in the left plot of Figure 3. In this scenario, it takes approximately 12 milliseconds to deliver power to all the loads and allows for the solar arrays to operate at the full load voltage, 117.8 volts, and delivers the requested $155 \mathrm{~kW}$ of power. This scheme allows for the power system to deliver the required power and voltage but does also allow high power and voltage swings during the 12 milliseconds power up phase.

An alternative solution is to increase the transition time from 1 millisecond to 20
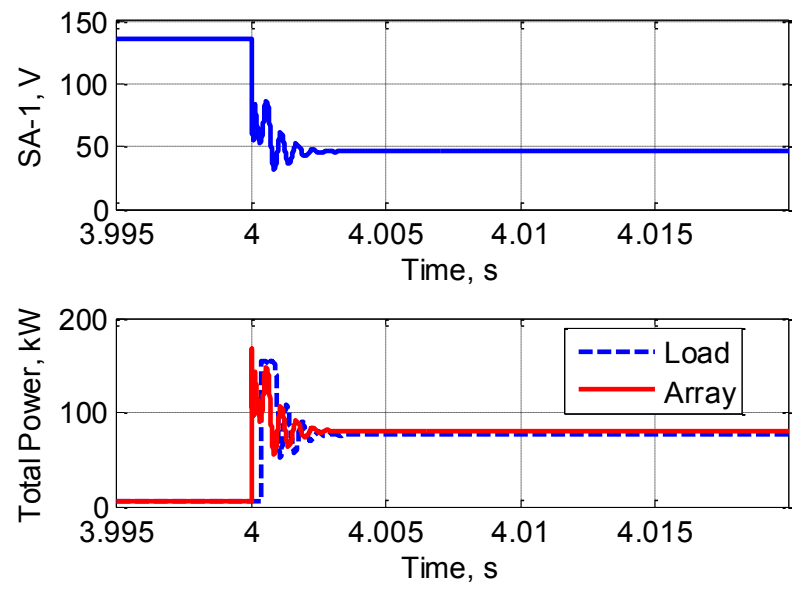

Figure 2. Solar array voltage response (top plot) and total array and load power (bottom) when turning all propulsion power strings on.

American Institute of Aeronautics and Astronautics 

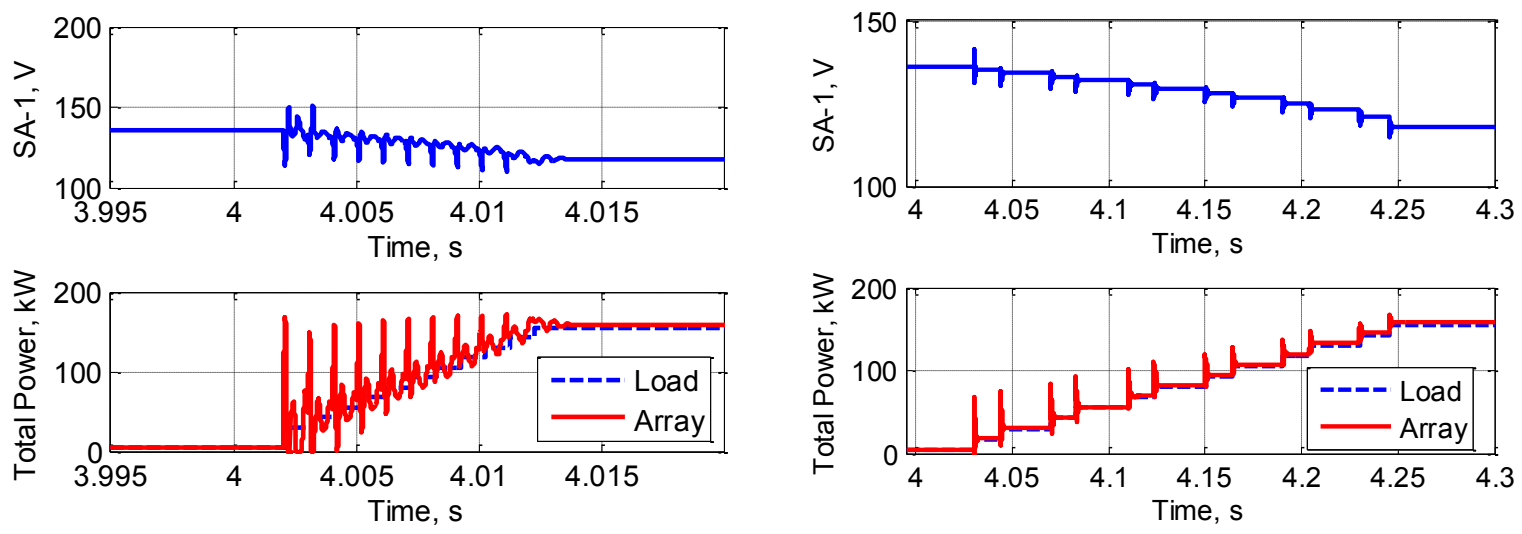

Figure 3. Solar array voltage response and total power, both load and array, when turning on each propulsion string with 1 millisecond between (left plot) and 20 milliseconds in between each command.

milliseconds. In this scenario, it would take 240 milliseconds to turn on all twelve loads which is still less than the 1 second update rate of the flight controller and therefore is acceptable. The response is shown in the right plot of Figure 3 and shows that the voltage and power swings (max and min) are smaller than the faster 1 millisecond update. Increasing the transient delay time to 20 milliseconds seems to provide a clear and more stable system response.

\section{B. Fault Response}

Another primary concern of the power system is how it responds to faults within the system. To test this, a short is simulated on the first power string (power string \#1) at a simulation time of 5 seconds. Figure 4 shows the response of the power generation and distribution to a short in power string 1 between the output of the RBI (shown as SW-1 of Figure 1) and the PPU. From the middle plot of Figure 4, which compares power string 1 and 7 (PS-1 and PS-7 respectively), at the simulation time of 5 seconds, the current of all the power strings becomes negative, current flowing in the opposite direction, and settles to 0 amps in about 1 millisecond. The solar array voltage decreases to approximately 16 volts until the RBI of power string 1 opens at 50 milliseconds to cut off the current to the faulted load. When the RBI opens, the power system recovers by restoring power to each of the remaining power strings, however the power system is operating at sub power, which is explained in more detail in the next section.

\section{Stability Analysis}

Stability analysis for the AEPS PPU can be addressed from two viewpoints - large signal and small signal. Large signal stability will be addressed first since these effects were already observed in Section A. One critical aspect in DC systems powered by solar arrays is the intersection of load lines. Figure 5 depicts the power/voltage curve for one solar array superimposed on the power/voltage curve for the six PPU loads (note that this can be doubled for the total non-segmented power system, and neglects the avionics and support system power). As can be seen, the two curves intersect at three different points, creating four "sections" of the solar array curve. In sections where the solar array power is greater than load power, the array is producing excess current, which will feed system capacitance and increase the voltage. In sections where the solar array power is less than the load power,
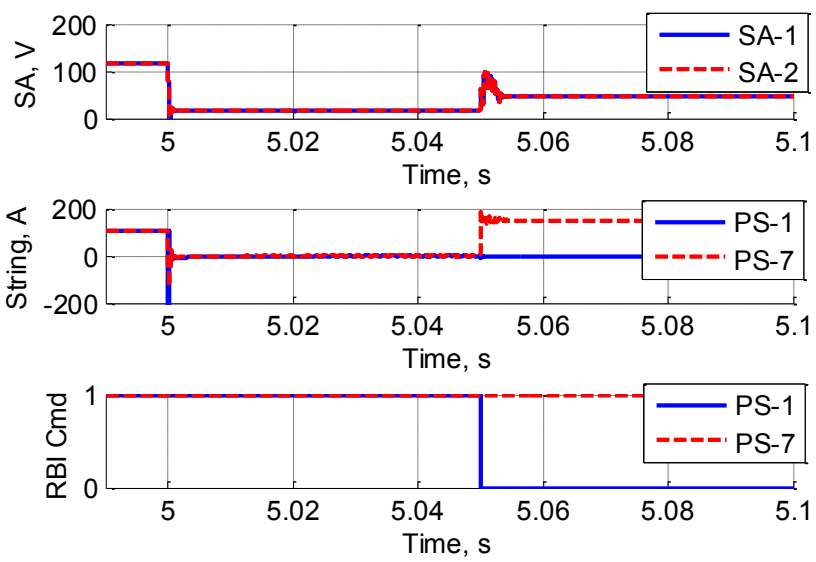

Figure 4. The solar array voltages (top), power string 1 and 7 (PS-1, PS-7 respectively) current (middle), and power string RBI command (bottom) during a fault (short) in power string 1 .

American Institute of Aeronautics and Astronautics 
current will be drawn from the system capacitance and decrease the voltage. Following this idea, arrows can be drawn in the diagram to indicate the direction of voltage change in each section. The intersection points of the array and load curves indicate equilibrium points, where the source and load powers are balanced. The equilibrium points with arrows pointing towards each other are stable, whereas intersection points with arrows pointing away from each other are unstable (since a small perturbation will move it further from the equilibrium point).

The simulation results in Figure 2 depict a scenario in which the transient (turning all strings at full load simultaneously) is large enough to push the bus voltage below the unstable equilibrium point, and it settles at the low-voltage/low-power stable equilibrium point. Note that the fault scenario in

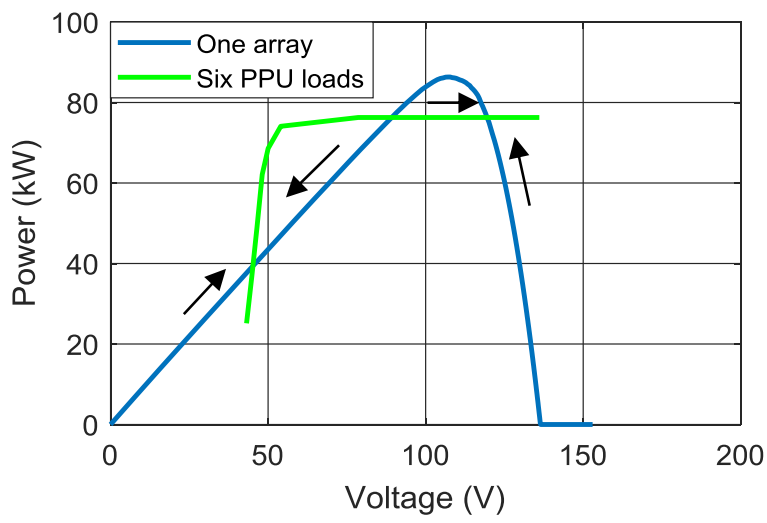

Figure 5. Power/voltage curves for solar array and PPU loads. Section B depicts a similar situation where the short pushes the bus voltage below the unstable equilibrium point and it settles to another low power voltage that is slightly higher. The diagram in Figure 6 depicts the bus voltage transient from Figure 2 (black line) superimposed on the solar array's power/voltage curve (extended into a third dimension with time). The gray line is the approximate location of the unstable equilibrium. As can be seen this point is exceeded during the transient, and the array voltage cannot recover to the high-voltage equilibrium. The bus voltage transient associated with the $20 \mathrm{~ms}$ load steps (seen in Figure 3) has been superimposed on the array power/voltage curve in Figure 7. As can be seen, for this case, the more gradual transient does not reach the critical voltage. Consequently, the bus voltage stabilizes to the desired equilibrium point. Adjusting the foldback points for the DDCU/load combination could eliminate the undesirable equilibrium points, and then the dynamic (small signal) stability of the desirable equilibrium point would be the only remaining concern.

PCKA's ISS model library also includes a tool to facilitate the calculation of eigenvalues for the linearized small signal system dynamics. This was applied to the non-segmented power system to evaluate dynamic stability of the system. The full system Jacobian matrix was calculated at 10 load levels in equal steps from $10 \%$ up to $100 \%$ for all 12 PPUs. Then, the eigenvalues of the system were calculated from the Jacobian matrices. Most eigenvalues do not change appreciably as a result of changing the load; however, the eigenvalues associated with the input filter capacitors on the DDCUs were found to change significantly (associating states with particular eigenvalues was done by calculating participation factors). The change in this particular eigenvalue due to the load level is depicted in Figure 8. Therein, it can be seen that the eigenvalues change from oscillatory (with non-zero imaginary parts) to purely real

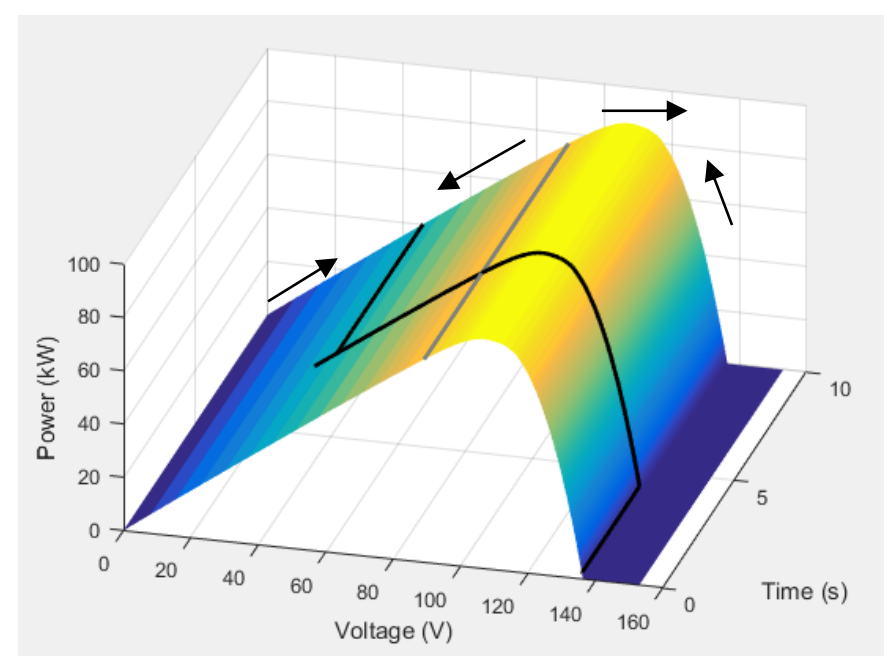

Figure 6. Step load transient of array power/voltage relationship. 


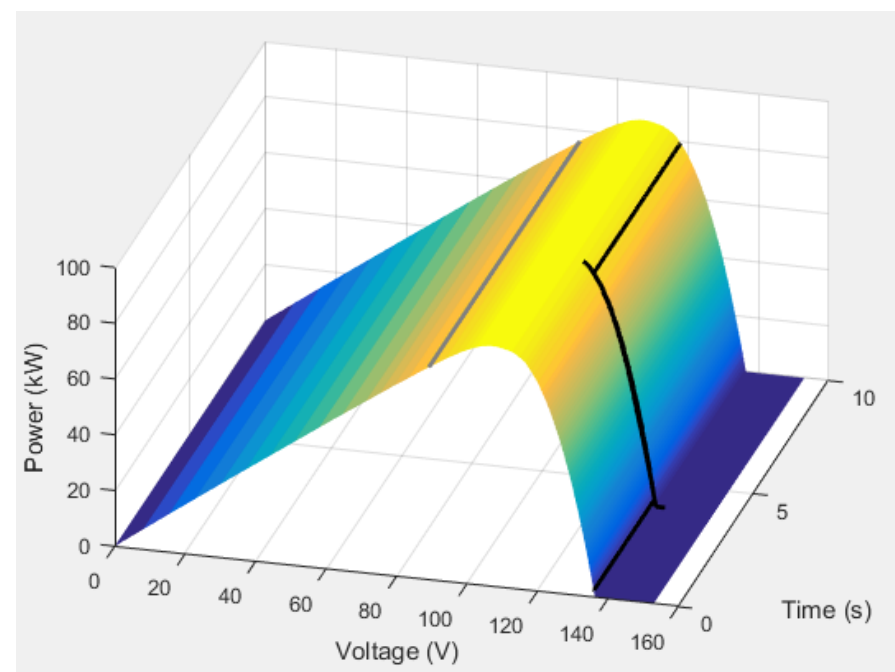

Figure 7 Sequential load transient of array power/voltage relationship.

and it moves toward the origin. This trajectory of eigenvalues is moving toward instability (positive real part); however, instability is not reached at full load. This indicates that dynamic stability is not a concern for the system as designed. The issues with large-signal stability observed with the unregulated systems can be largely avoided in the regulated case. The SSU can maintain the array at a high-current voltage level and quickly route the shunted current to loads during a load step-on transient. As long as the total load does not exceed the peak power of the arrays, the voltage can be maintained above the critical point corresponding to the unstable equilibrium point. The effects of increasing load on the small signal stability were also examined. The black markers in Figure 8 show the equivalent eigenvalues for $\mathrm{C} 2$ for the regulated case. With the bus voltage held nearly fixed by the regulators, these eigenvalues do not move nearly as much as the unregulated case. It was found that eigenvalues involving the SSU controller and the terminal capacitor (C1) do change significantly. They are depicted in Figure 9. These eigenvalues do move toward the imaginary axis, but as above, do not cross, so the system remains stable.

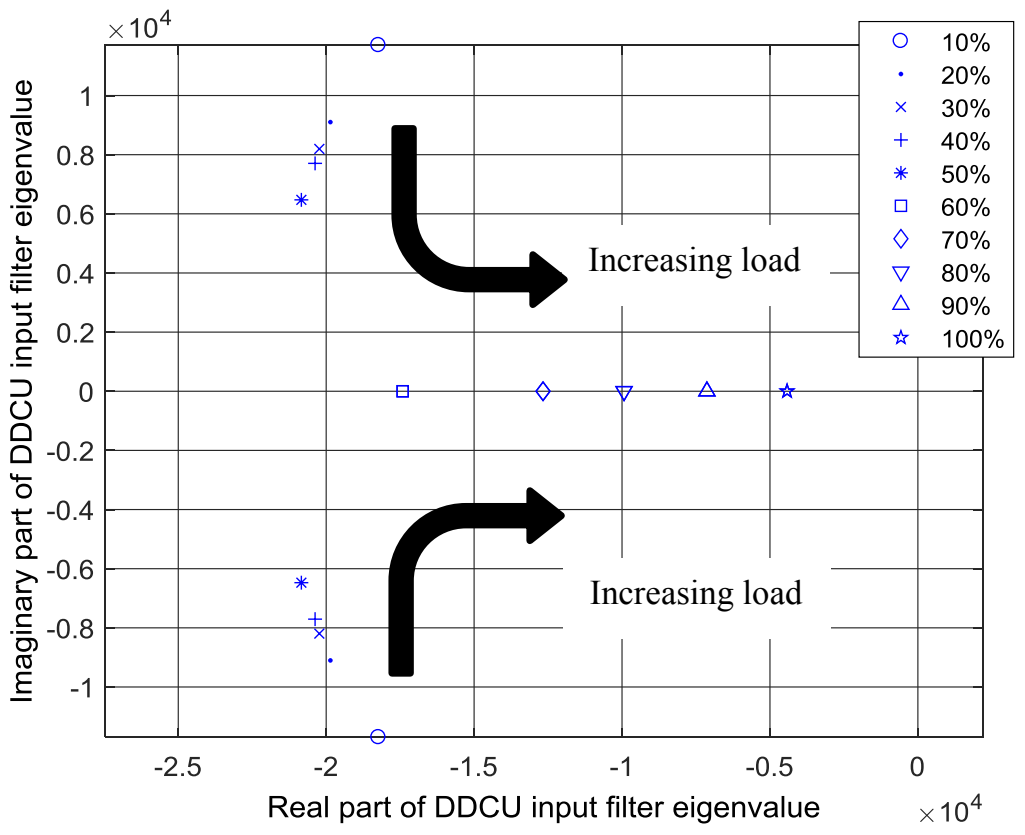

Figure 8 DDCU input filter eigenvalue sensitivity to load power. 


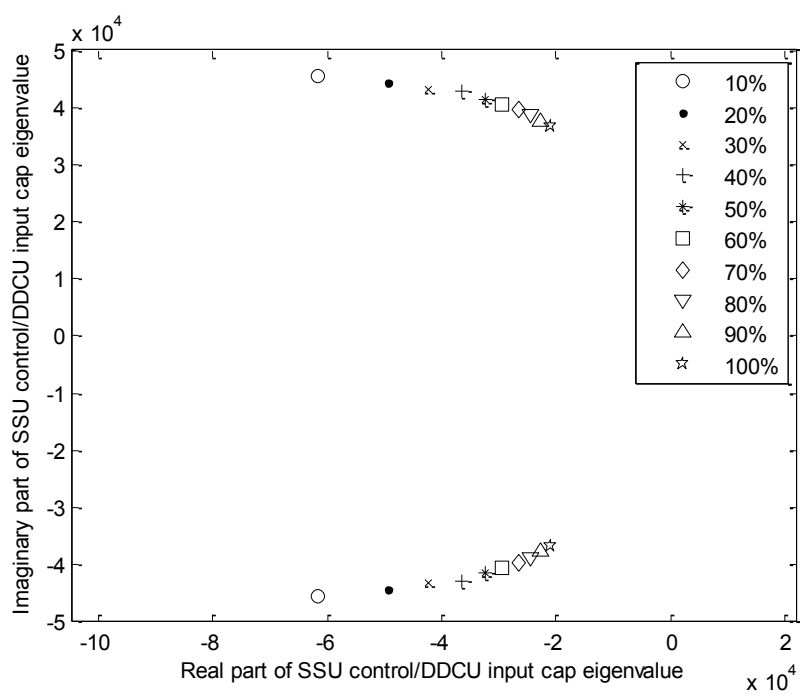

Figure 9. SSU control eigenvalue sensitivity to load power.

\section{150kW Segmented Power System}

The second $150 \mathrm{~kW}$ architecture is a segmented power system consisting of 12 electric propulsion strings consuming $12.5 \mathrm{~kW}$ of power each with 2 additional branches dedicated for $2.5 \mathrm{~kW}$ of power each for avionics and other support systems. This architecture features a segmented bus, where six electrical branches and one support branch are powered by a single solar array to provide $77.5 \mathrm{~kW}$ of power each as shown in Figure 10. The complete power system is designed to provide $155 \mathrm{~kW}$ of power. At full power each solar array provides $79.4 \mathrm{~kW}$ of power, which is equivalent to the non-segmented architecture. In steady-state with no faults, the primary difference between the segmented and non-segmented architectures is the power available on the bus, with $155 \mathrm{~kW}$ for the non-segmented and $77.5 \mathrm{~kW}$ on two busses for the segmented architecture. This architecture could represent a more practical implementation as the bus current levels for the PDUs are effectively halved.

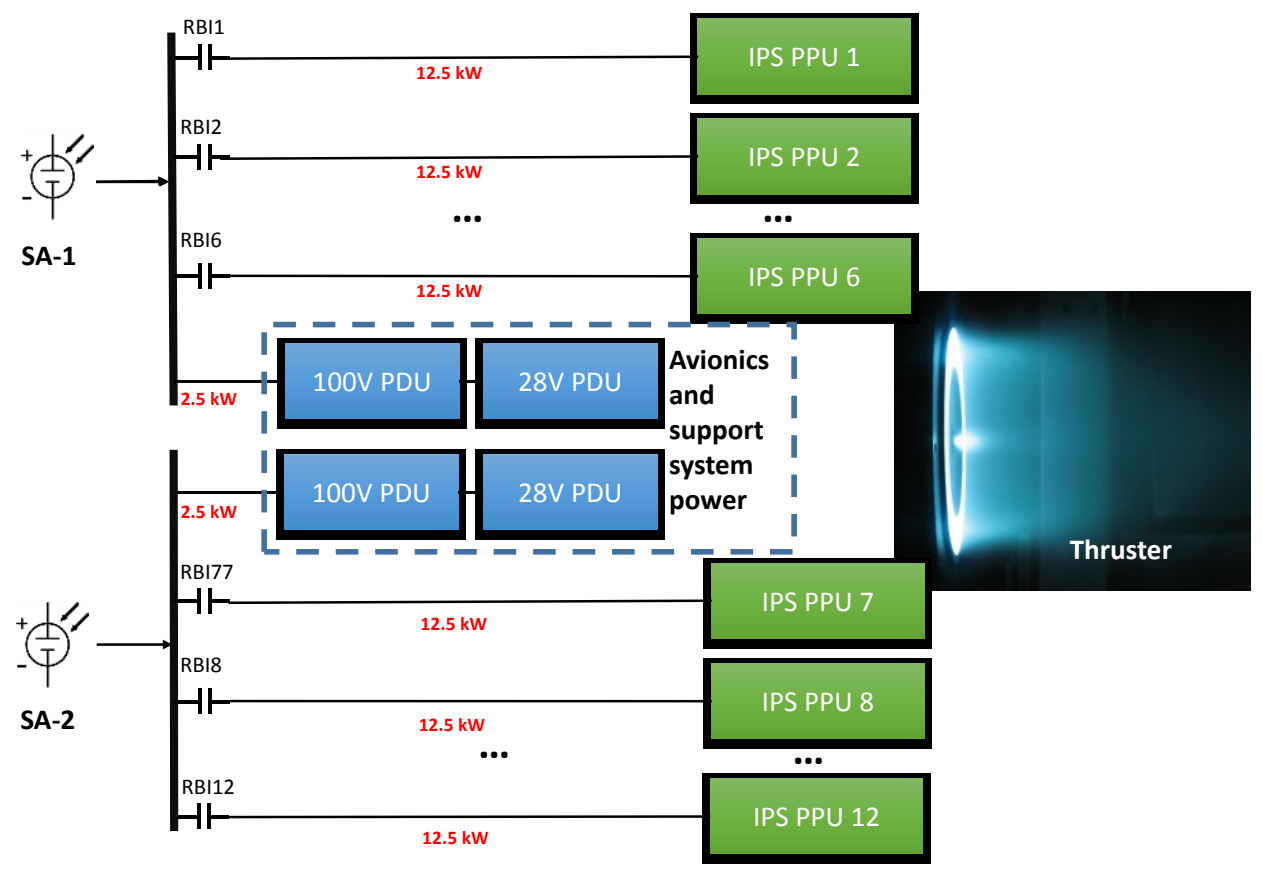

Figure 10. The 150kW segmented architecture.

American Institute of Aeronautics and Astronautics 


\section{A. Transient Response}

The transient response of the segmented power system when turning on all loads at one time is shown in Figure 11 and is identical to the non-segmented power system response shown in Figure 2. In this instance, there is no difference in the total power demand from the loads or total power supplied from the source. Next, both a 1 millisecond and 20 millisecond delay are inserted between turning on each power string. The response with a 1 millisecond delay is shown in the left plot of Figure 12 and the 20 millisecond delay in the right plot of Figure 12. Figure 12 shows that adding the delay in the commands cleans up the signal, which is the same trend as shown with the non-segmented power architecture and shown in Figure 4. However, comparing the segmented (Figure 12) to the nonsegmented (Figure 4) architectures, the segmented power architecture has smaller min and max voltage and power values when turning on each load. For the segmented case, turning on load \#7, which is the first load attached to the second solar array, does result in higher min and max values than turning on the $7^{\text {th }}$ power string of the non-segmented. This is because the $7^{\text {th }}$ string of the segmented architecture is the first load attached to the second solar array and will more closely resemble turning on load 1 of the segmented architecture. The other option would have been to trade between the two solar arrays as the loads were turned on and in this case the larger increase would not have been seen later in the sequence.
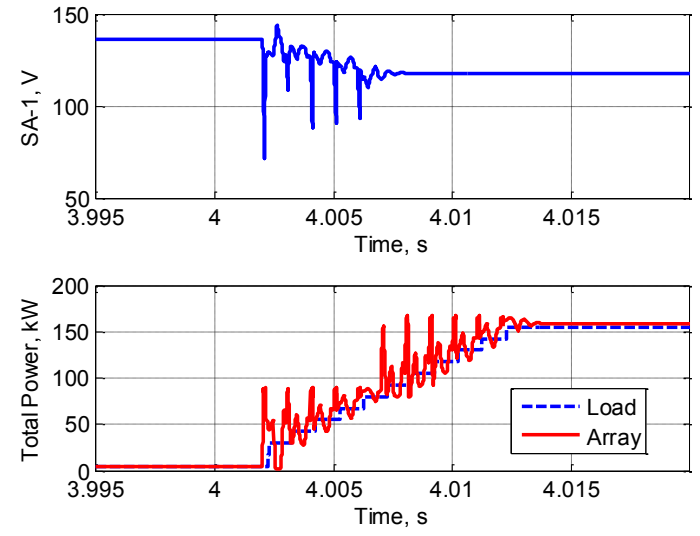

a)
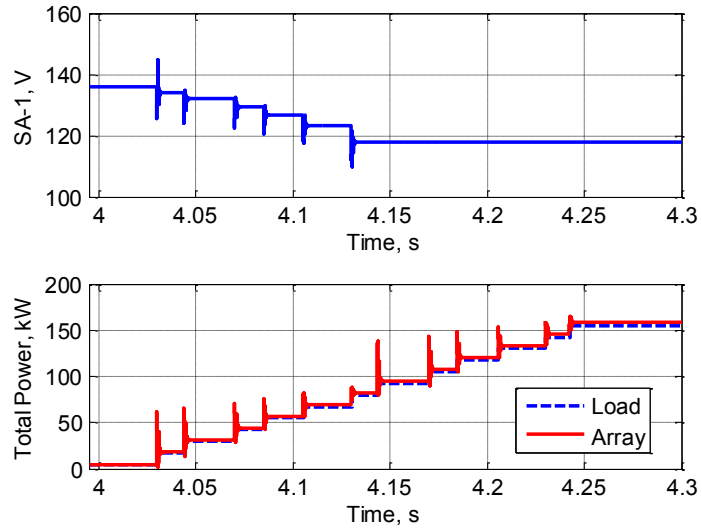

b)

Figure 12. Solar array voltage and total power for the segmented power architecture with the loads turned on in increments of a) 1 millisecond (left) and b) 20 milliseconds (right) between commands. All loads connected to bus 1 are turned on before turning on the loads connected to bus 2 .

\section{B. Fault Response}

As with the non-segmented architecture, to test the fault response of the system a short is simulated on the first power string (power string \#1) at a simulation time of 5 seconds. For the segmented architecture, power strings 1 through 6 are attached to bus 1 and solar array 1, while power strings 7 through 12 are attached to bus 2 and solar array 2 . The voltages and currents associated with bus 1 decrease to zero when the short is inserted and remain at zero 
until RBI-1 opens and cuts off power, allowing the voltage to increase. The voltages and currents associated with bus 2 remain untouched and still provide power to the propulsion system and support systems. With this architecture, the system is able to restore the voltage on the remaining operational loads connected to bus 1 (loads 2 through 6) to the same voltage as before the fault. In this architecture, the fault does not cause the bus voltage to drop below the critical voltage. After the fault, there is less total current draw from the solar array, therefore solar array 1 will operate with a slightly higher voltage than solar array 2.

\section{Stability Analysis}

From a mathematical perspective, the stability analysis for the segmented and non-segmented systems is identical; therefore, the results in Section II-C apply to the segmented system as well.
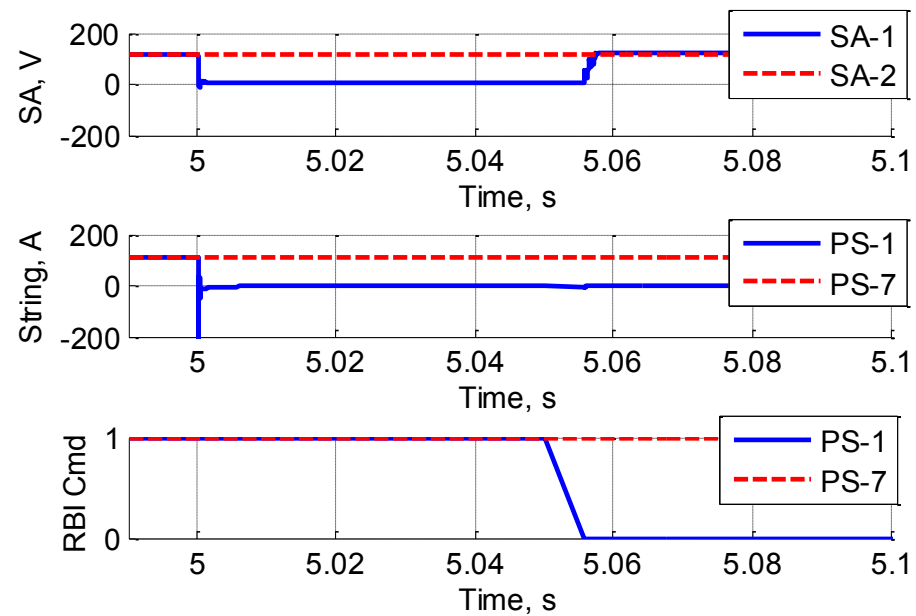

Figure 13. Solar array voltages (top), power string current (middle), and RBI commands (bottom) for the segmented power architecture.

\section{Regulated Architectures}

A sequential shunt unit (SSU) can be added at the output of each solar array to provide a more stabilized output voltage and power output. The SSU block is part of the ISS Simulink library toolbox and represents the behavior of a sequential shut unit. The SSU regulates the bus voltage by supplying a resistive load which increases as the load decreases to provide a constant voltage and load as done on the ISS. ${ }^{4}$ Figure 14 shows the solar array voltage and total power of the a) non-segmented and b) segmented power architectures. With the SSU included in the power system, the total power and solar array voltage is more consistent and has much smaller swings as the loads are turned on, as shown in Figure 14.
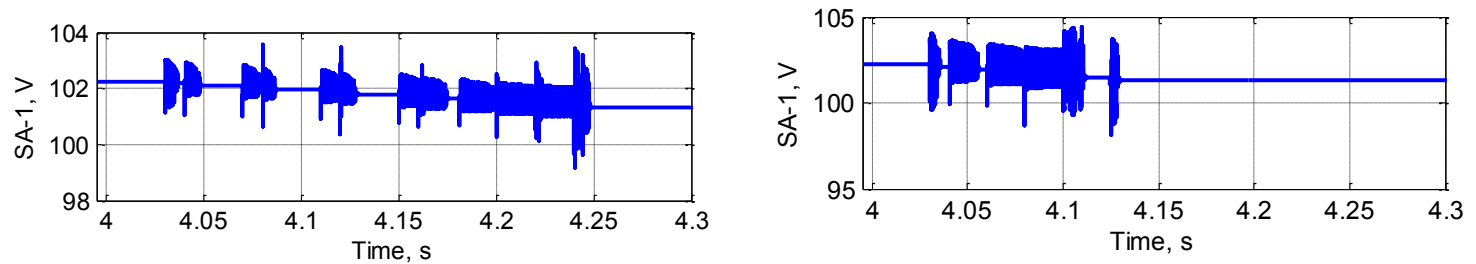

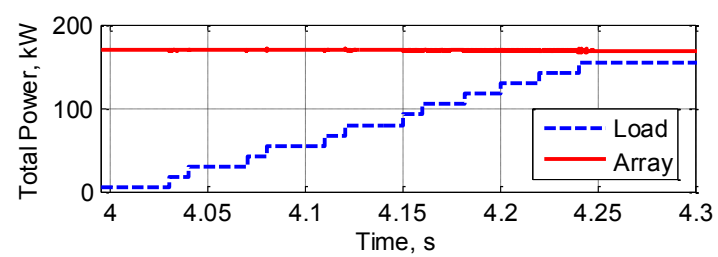

a)

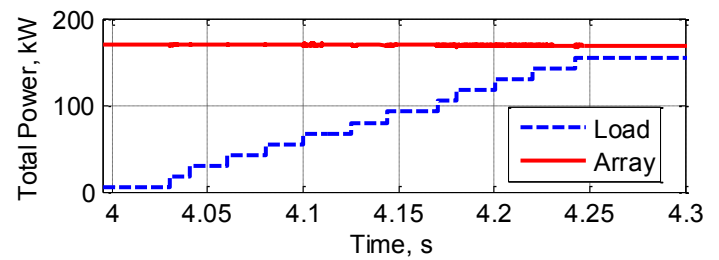

b)

Figure 14. The solar array voltage and total power for the a) regulated non-segmented power architecture (left) and b) the regulated segmented power architecture (right) to turning on all loads in 20 millisecond increments. 
Figure 15 compares the minimum and maximum voltages obtained when turning on an additional load and the steady-state value obtained when the voltage settles. The top plots show the non-segmented power architecture while the bottom plots show the segmented. The left plot (a) shows the systems without the SSU while the right plots (b) show the results with the SSU at the output of each solar array. In the segmented case with no SSU, all the loads connected to string 1 (solar array 1) are turned on before powering any loads attached to string 2 , which is the reason why the lowest minimum voltages are obtained when turning on loads 6 and 12 (last loads of each string) and why turning on loads 1 and 7 results in the largest maximum voltage (first of each string).
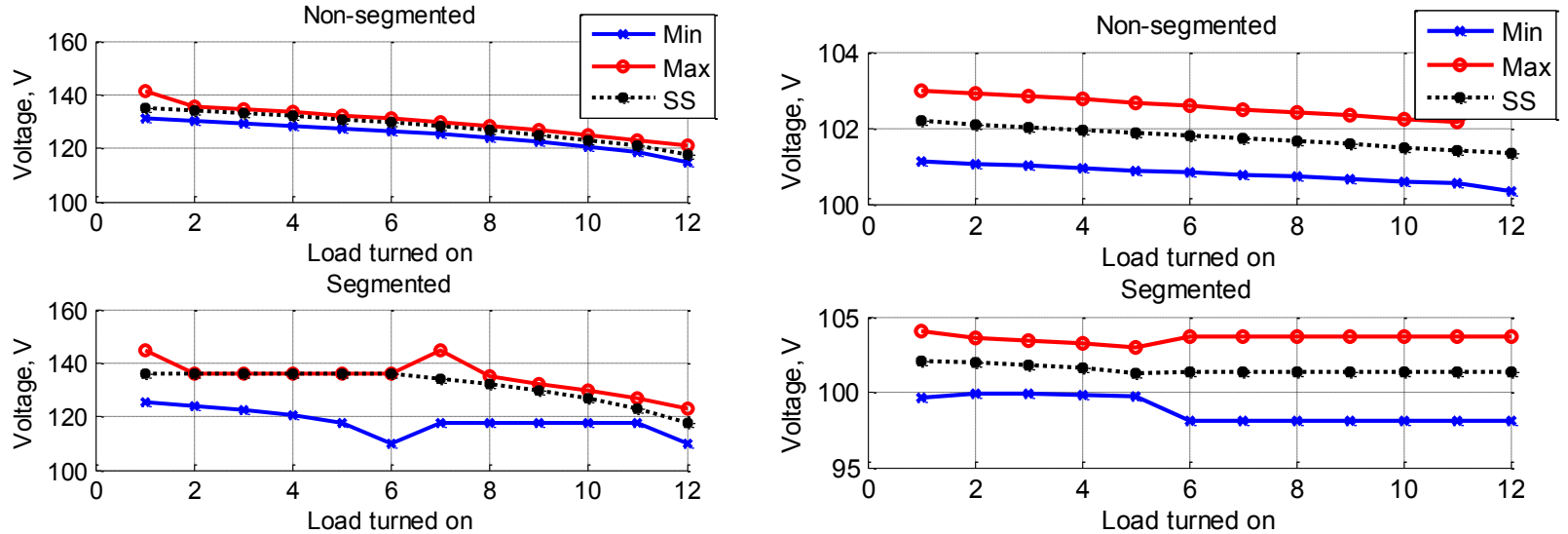

a)

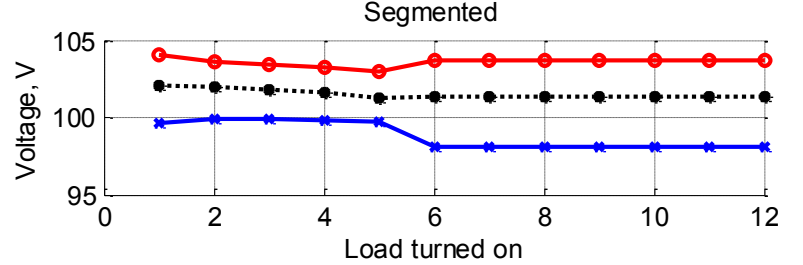

b)

Figure 15. The minimum, maximum, and steady-state voltages when turning on each load. The left plots a) show the non-segmented (top) and segmented (bottom) with no SSU, while the right plots, b) shows the voltages with the SSU included.

The issues with large-signal stability observed with the unregulated systems can be largely avoided in the regulated case. The SSU can maintain the array at a high-current voltage level and quickly route the shunted current to loads during a load step-on transient. As long as the total load does not exceed the peak power of the arrays, the voltage can be maintained above the critical point corresponding to the unstable equilibrium point. A fast-switching regulator can also be an effective tool in stabilizing the small signal behavior of the system.

\section{Discussion/Comparison}

This paper looked at four different $150 \mathrm{~kW}$ power systems, non-segmented, segmented, non-segmented regulated, and segmented regulated. In each case, the numerical stability analysis provided the same results and will not be discussed further in this section. The solar array voltage and power supplied to the loads as a function of time when turning all loads on is shown in Figure 16. As shown in Figure 16 and in the prior sections, the regulated architecture with the SSU unit provides a more constant power and voltage on the bus and removes the large power swings seen when additional loads are added to the bus. This includes transient changes in the power demand from the loads. As for the common versus segmented bus architecture with the power being regulated, there is not much difference in the performance. The one advantage that the segmented architecture does provide is in a fault scenario on the power generation side, such as a solar array failure, where all strings would not draw power from the remaining solar array. In a segmented case, half the power strings would lose power and the other half would provide nominal power. This is important when considering that avionics and life support systems need continuous power.

American Institute of Aeronautics and Astronautics 

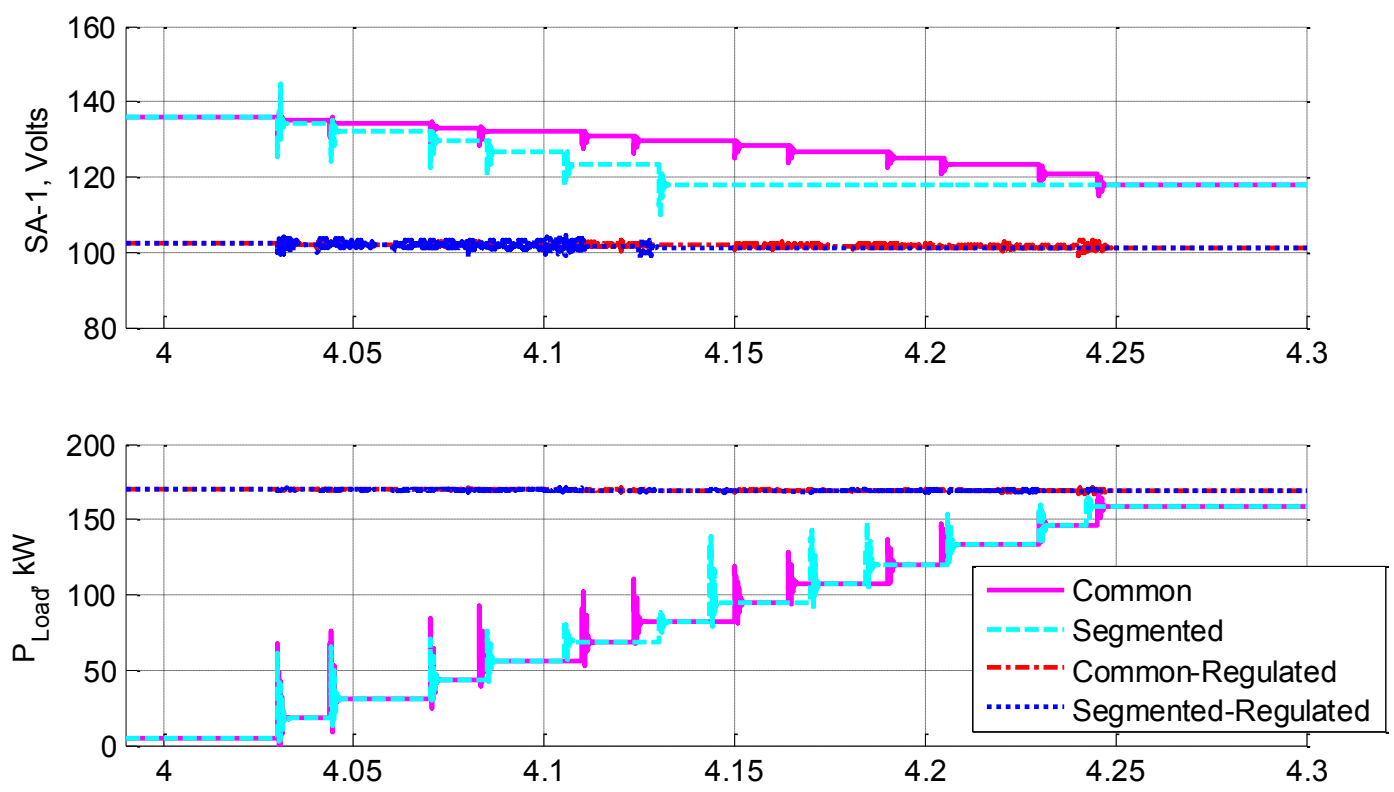

Figure 16. The solar array 1 (SA-1) voltage (top) and load power (bottom) as a function of time when turning all loads on with a small delay added.

\section{Conclusion}

NASA, in conjunction with PC Krause and Associates, has created and analyzed a MATLAB ${ }^{\circledR} /$ Simulink based model of a $150 \mathrm{~kW}$ power architecture, which is based off the $50 \mathrm{~kW}$ class Government reference design of the Solar Electric Propulsion Module (SEPM) on ARRM. Flight versions of these electric propulsion strings are being developed under the NASA SEP TDM-led Advanced Electric Propulsion System (AEPS) Project. This paper compared four different architectures. The first architecture contains two solar arrays connected to a single bus which distributes power to twelve propulsion strings and two avionics and support strings. The second architecture is a segmented system in which a solar array powers a single bus which powers six power strings and a single avionics and support system string. The remaining two architectures are regulated versions of the first two architectures where a sequential shunt unit is added between the solar array and the bus to regulate the voltage to the bus by adding a simulated load to the system. This paper showed, through simulation results with an analytical analysis, that the segmented architecture has an improved fault response to a short in one of the loads. With the segmented architecture, when a short is added to one of the propulsion strings, the short did not decrease the bus voltage beyond the critical stability voltage and therefore had the ability to restore the desired voltage to the remaining lines, whereas the non-segmented architecture was not able to provide the desired voltage to the remaining loads after the fault due to the bus voltage decreasing beyond the critical voltage. The addition of a sequential shunt unit regulated the bus voltage, in steady-state, and helped reduce the power and voltage oscillations seen when turning on loads, providing more consistent voltage regardless of which loads were turned on.

\section{References}

${ }^{1}$ Herman, D.A, Santiago, W., Kamhawi, H., Polk, J.E., Snyder, J.S., Hofer, R.R., and Sekerak, M.J., "The Ion Propulsion System for the Asteroid Redirect Robotic Mission," AIAA 2016-4824, 52 ${ }^{\text {nd }}$ AIAA/SAE/ASEE Joint Propulsion Conference, Salt Lake City, UT, July 25-27, 2016.

American Institute of Aeronautics and Astronautics 
${ }^{2}$ Loop, B., "Simulation Environment for Power Management and Distribution Development," SBIR Technologies Workshop, Denver, CO, June 28, 2012.

${ }^{3}$ White, R.V., "Fault Tolerance in Distributed Power Systems," IEEE, International Conference on Power Electronics (CIEP), MEXICO, San Luis, Potosi, October 16-19, 1995.

${ }^{4}$ Wikipedia Contributors, "Electrical system of the International Space Station," Wikipedia, The Free Encyclopedia, https://en.wikipedia.org/wiki/Electrical_system_of the_International_Space_Station\#SSU, assessed March 30, 2017. ${ }^{5}$ Gerstenmaier, W.H., "Progress in Defining the Deep Space Gateway and Transportation Plan," Presentation to the NASA Advisory Council, March 28, 2017. 